\title{
Ernan Haruvy
}

University of Texas at Dallas

Vijay Mahajan

University of Texas at Austin and Indian School of Business,

Hyderabad

\section{Ashutosh Prasad}

University of Texas at Dallas

\section{The Effect of Piracy on the Market Penetration of Subscription Software*}

\section{Introduction}

Software piracy refers to the illegal use of software by end users. The Business Software Alliance (BSA) in its 2002 annual report states that $40 \%$ of business software installed on computers is pirated, resulting in $\$ 11$ billion in losses (BSA 2002). Given that piracy is ubiquitous in software markets, managers need to consider it in their marketing decisions. While piracy on a large scale is clearly detrimental to profits, a strategic tolerance toward some piracy may actually increase profits. Specifically, piracy may help in diffusing the software to its target market because it increases the size of the user base, resulting in benefits to legal buyers due to network externalities.

A network externality is the additional utility derived from the size of the user base (Katz and Shapiro

* We thank Sridhar Balasubramanian, Nanda Kumar, Stan Liebowitz, Preston McAfee, Ram Rao, seminar participants at the University of Texas at Dallas, the editor, and three anonymous referees for their valuable input. Contact the corresponding author, Ashutosh Prasad, at aprasad@utdallas.edu.

(Journal of Business, 2004, vol. 77, no. 2, pt. 2)

(C) 2004 by The University of Chicago. All rights reserved. 0021-9398/2004/7702S2-0005\$10.00

Software piracy is not necessarily harmful to a software firm seeking to launch a new product. When strategically managed through the use of price and protection measures, piracy establishes the initial adopters of the software, who in turn influence other users to buy the product. We examine the role of piracy in affecting the adoption of subscription software products. We present an individual-level model in an adaptive population setting intended to determine the price-protection mix that maximizes the discounted profit stream over the life of the software. An extension for nonsubscription software is also discussed. 
1985). Software products, like many other products (e.g., telephones and fax machines), have the feature that they are more useful when other people have them as well. A larger software network size provides greater utility to consumers due to, among other things, file compatibility and transferability, reduced uncertainty about product quality, a larger base of compatible software, and better availability of books, magazines, newsgroups, and services.

Network externalities play an important role in determining the success of new software. The marketing challenge in launching new software is that, being new, the software does not have an established user base and is therefore less attractive to users, and not being able to attract more users, it is difficult to establish a user base (Farrell and Saloner 1986). Piracy can help in establishing the initial user base, but it has to be limited through appropriate pricing and piracy protection.

By piracy protection we mean all antipiracy actions by the firm that lower expected value to pirates from the software. An assumption in this work is that protection can be managed through software and hardware design. Some software firms provide documentation that is difficult or impossible to photocopy and software codes that limit usage if the software is not registered. Certificates of authenticity, holograms, and incorporating passwords are other such devices. As a recent example, by implementing an activation feature in its latest operating system, Windows XP, Microsoft hopes to severely curtail piracy of that product (Bevan 2001). Furthermore, pirates will not receive many of the service and upgrade benefits that legal buyers obtain. Other attempts to deter piracy include the use of noncommercial disk sizes that are difficult to duplicate. The advent of the Internet has allowed individual copies of a software product to send messages to the firm with an ID and an IP (Internet protocol) address, allowing a firm to track each copy of its product each time it is used.

Givon, Mahajan, and Muller (1997) conjectured that the market success of Excel over Lotus 1-2-3 may have been due to its higher tolerance for piracy. Other such practices are not hard to find. Unlike Microsoft, Apple does not have copy protection on its latest MacOS (Williams 2002). Software by smartesolutions.com—with the tagline "managing piracy for profits"-allows software to incorporate a set reduction in quality that will "convert unlicensed users into paying customers." Many firms have the ability to track piracy through online registration, but make it optional (e.g., Adobe Acrobat; Messitt 2002). Trial software and shareware versions that have a specified expiration date often do not disable all features following expiration and instead either disable selected features or display lengthy (and time-consuming) warning messages. ${ }^{1}$ Three such popular trial software versions are SnagIt (advanced screen capture), WinZip (file compression), and BrainForest Mobile Edition

1. Software products that are time limited are pirated only if they continue to be used after the trial time expires and if the terms of the license prohibit such use. Before the time of expiry, they would not be examples of piracy. One may call them freeware or shareware before the trial time expires. 
(project planner). Gaming sites that do not frequently change their entry passwords and tolerate the exchange of passwords on usenet groups are yet other examples.

Price also influences piracy (Nascimento and Vanhonacker 1988; Conner and Rumelt 1991; Takeyama 1994). At a high price, buying the product becomes less attractive relative to pirating it; at a low price, the reverse is the case. Though a lower price is expected to result in reduced piracy, a lower price also translates into lower profit margins. These considerations imply that price has to be determined simultaneously with protection to maximize profits.

Using evolutionary dynamics, we investigate the implications of piracy for newly launched software. We find that a moderate tolerance for piracy is useful in facilitating both faster adoption and higher prices. There are conditions that tend to make piracy less useful, most notably when piracy control is costly, information is precise, penetration is quick, externalities are low, future profits are greatly discounted, customer inertia is low, or product life is short. Then tolerance for piracy is not recommended.

Our analysis deals primarily with subscription software, that is, software for which the customer must periodically pay a subscription or license fee. With subscription software the firm only sells a license to use the software for a limited period of time, and the license must be continually renewed. This seems to be a trend in the software industry, and piracy in this context is common. Among other things, users may use the software without a license or continue to try to operate the software after expiry of the license. The software publisher is free to allow this or, through time stamping, it can reduce features or the operation of the software altogether after the expiry period. For example, the statistical software SAS comes with a 12-month subscription, after which a user (legal or pirate) must obtain a SETINIT disk every year. In other cases, a pirate may "hack" into the system by bypassing a security mechanism.

As other examples of subscription software that have been pirated, the BSA, in a recent operation called Operation Bidder Beware, tracked the sale of pirated software from vendors in the United Kingdom, Germany, and the United States (BSA 2000). The BSA identified the offending vendors through the purchase of software products advertised on auction sites at a fraction of their retail price. After examination of the evidence, BSA filed civil actions in federal court in the United States. Among the pirated and counterfeit programs purchased by BSA were products published by Adobe, Autodesk, Network Associates, and Symantec. Autodesk products are offered on a subscription basis. Symantec is the maker of Norton Antivirus, which is sold as a subscription (typically with a purchase, a user will get a 12-month subscription). Network Associates is the maker of McAfee Antivirus and offers subscriptions to its popular VirusScan Online, Personal Firewall Plus, and Privacy Service. Symantec and Network Associates provide insight into how piracy, often done through copying disks, relates to subscription that is Web based. In the case of Symantec, a sold disk comes with a 12-month subscription 
after which subscription services must be obtained. Hence, a pirate wishing to "renew" the subscription must continually copy disks.

Subscription markets have been receiving greater attention in recent years with regard to brand switching (e.g., Fudenberg and Tirole 1997), behaviorbased price discrimination (e.g., Chen 1997), telephony (e.g., Keller 1997), banking (e.g., Baily and Kilman 1998), and electronic commerce. Subscription products are a significant component of the software market. Three large software firms have recently launched initiatives with this in mind. Microsoft has recently announced its new focus on the Microsoft.net initiative to make existing and new software available on the Web on a subscription basis. Oracle has similarly launched portal.oracle.com, and Sun Microsystems has launched Destination ASP, a program to advocate the service provider model and to provide service providers with tools. In addition to the software firms themselves, independent Internet businesses known as application service providers (ASPs) offer Microsoft and other software for subscription on a monthly basis. Examples include digex.com, always-on.com, and personable.com. Gurnami and Karlapalem (2001) suggest that existing models of firm strategic decision making are inappropriate for increasingly prominent Internet-based software dissemination. The current analysis is intended to fill this gap.

The rest of the article is organized as follows: Section II examines relevant literature and highlights the relative contribution of the proposed model. In Section III we discuss the basic model for subscription software and provide static analysis. In Section IV we develop the dynamic model and analysis, and provide an illustrative example. Section $\mathrm{V}$ extends the model to nonsubscription software and evaluates this model on empirical data. Section VI concludes with a discussion and suggestions for future research directions.

\section{Background Literature}

Two distinct streams of literature consider the issue of piracy in software adoption: the literature on diffusion modeling (Nascimento and Vanhonacker 1988; Givon, Mahajan, and Muller 1995, 1997; Prasad and Mahajan 2003) and the literature on network externalities (Conner and Rumelt 1991; Takeyama 1994). These studies allow potential adopters the three choices of Buy, Pirate, or Not Adopt instead of the typical two (Buy, Not Adopt).

In contrast to the model proposed here, which is at the individual level, the diffusion models of piracy are at the aggregate level. The advantage of individual level, or utility-based, models over aggregate-level models is that it is easier to incorporate decision variables. Though the variables here are price and protection, numerous other product and individual characteristics could be incorporated under this framework. We note that several prominent works (e.g., Stoneman 1981; Jensen 1982; Oren and Schwartz 1988; Lattin and Roberts 1989; and Chatterjee and Eliashberg 1990) have focused on the individual decision maker in the context of diffusion. However, these articles 
do not consider piracy or network externalities that are important characteristics of software markets.

In the literature on piracy using network externalities in a utility-based framework, the treatment of customer expectations on the network size has been somewhat problematic. Consumers are assumed to have perfect foresight about the final outcome instantly upon introduction and to behave in accordance with that outcome. We adopt an alternative approach in which aggregate demand is derived from individual level adaptive models, specifically dynamic logit (e.g., Chintagunta and Rao 1996; Van den Bulte and Lilien 1999). Equally important from a manager's perspective is that future profits are discounted to present value. Hence the dynamics of adoption matters.

Characterizing the dynamic adoption process is also helpful in settings characterized by network externalities, as these settings are often plagued by multiple equilibria (Katz and Shapiro 1985). A specification of dynamics resolves the multiplicity problem without the need for common ad hoc assumptions such as collective rationality-the idea that if all agents prefer one outcome to other outcomes, it will be selected (Van Huyck, Battalio, and Beil 1990; Crawford 1991, 1995; Goeree and Holt 1999). Thus, the proposed model provides some unique contributions to the literature on network externalities.

As a side note, other means to exploit network externalities are available to the software manager. These include (i) compatibility with existing products and cloning, where the new software tries to benefit from the established network of other software; (ii) seeding or sampling, in which free copies of the software are made available to influential users (Jain, Mahajan, and Muller 1995); (iii) freeware and shareware, where a part of the new software is given away free to signal the attractiveness of the commercially sold version (e.g., Padmanabhan, Rajiv, and Srinivasan 1997); and (iv) penetration pricing (e.g., Dhebar and Oren 1985), where the price of the software is initially low to attract more buyers and establish the network, and is later raised. The proposed method of piracy is a viable alternative to these methods when the firm wants a cheaper method (as opposed to designing or licensing compatibility) or has less information on the total market size and end users (as opposed to seeding). Even if another approach is used, piracy will be present, and therefore these methods are not independent of a piracy-based analysis.

\section{The Model}

The framework developed here is concerned with (i) the investigation of piracy as a mechanism for the exploitation of network externalities, (ii) the description of the adoption pattern, and (iii) the ability to jointly optimize with respect to both price and protection level. The firm's objective is to maximize its profit stream with respect to the decision variables, price, and protection. The sequence of actions is as follows: prior to the release of the product, the firm sets a protection level for the software and incurs a cost that is increasing in 
the level of protection. Since protection is feature related, it is finalized prior to the release of the product. The price, however, can be either set once or determined in each period depending on the setting being modeled. In each period for the life of the software, individuals decide whether to buy, pirate, or not adopt the software in that period. The assumption that the individual consumer makes a decision at the end of each period implies that the legal version expires at the end of each period unless a fee is paid. However, it does not imply that pirates lose access to the pirated software at the end of each period. If a pirate decides to continue using the pirated version in the next period, this is treated as a decision to pirate in the next period. ${ }^{2}$

A protection parameter $\alpha \in[0,1]$ denotes the protection level. If set to $\alpha=1$, the software is said to be fully protected, implying that a pirate derives no utility from the pirated version. If $\alpha=0$, the software is not at all protected, implying that a pirate will derive a utility from the pirated version equal to that of a legal buyer. The piracy protection requires disabling features in the product that reduce utility. It may be possible to distinguish between two types of utility reducing features: (1) product-intrinsic features and (2) externality-related features. The latter involves features relating to communication with other users, such as file sharing; access to user groups; chat rooms; the ability to play games with other users online, with many computer games now having this option; and the ability to send e-mails to other users, which is particularly important in matching services. We capture the distinction by denoting a reduction in the first set of features by $\alpha_{1}$ and the latter by $\alpha_{2}$. The cost parameter is $\theta$, similarly partitioned. The firm's objective is

$$
\max _{\theta_{1}, \theta_{2}, P(1), P(2), \ldots, P(T)}-\theta_{1} \alpha_{1}^{2}-\theta_{2} \alpha_{2}^{2}+\sum_{t=0}^{T} \delta^{t}[P(t)-M] n_{b}(t),
$$

where $\delta$ is the discount factor, $T$ is the expected life of the software, $P(t)$ is the subscription price in period $t$, and $n_{b}(t)$ is the number of buyers at time $t$. In the remaining analysis, with no loss of generality, the constant marginal cost $M$ is set to zero.

An individual's utility, or payoff, from the decision to buy, pirate, or not adopt the software depends on the valuation of the software, its installed base, its price, and its protection level. The utility vector $\mathbf{U}(\mathbf{t})$ gives the utility from each choice as a function of the installed base in period $t$ :

$$
\mathbf{U}(\mathbf{t})=\left(\begin{array}{c}
U_{\text {Buy }}(t) \\
U_{\text {Pirate }}(t) \\
U_{\text {Not Adopt }}(t)
\end{array}\right)=\left(\begin{array}{c}
A+B\left[n_{b}(t)+\phi\left(\alpha_{2}\right) n_{p}(t)\right]-P(t) \\
A\left(1-\alpha_{1}\right)+B\left[n_{b}(t)+\phi\left(\alpha_{2}\right) n_{p}(t)\right]\left(1-\alpha_{2}\right) \\
V
\end{array}\right),
$$

2. In some cases, the pirate's access may physically expire at the end of the period. This does not affect the modeling, but it does make the pirate's decision to continue pirating more visible. 
where

$U_{j}(t)$ is the payoff to making choice $j$ in period $t$, where $j \in$ \{Buy, Pirate, Not Adopt\},

$A$ is the consumer valuation of the software if it has no installed base, that is, its intrinsic value,

$B$ is the incremental increase in product utility due to the addition of a buyer to the network,

$P(t)$ is the period $t$ subscription price of the software,

$V$ is the net value the consumer assigns to the best outside alternative to the software, which may include doing nothing,

$n_{b}(t)$ is the number of buyers of the product in period $t$,

$n_{p}(t)$ is the number of pirates of the product in period $t$,

$n_{n}(t)$ is the number of nonusers of the product in period $t$,

$N$ is the total size of the potential consumer population $N=n_{b}(t)+$ $n_{p}(t)+n_{n}(t)$,

$\alpha_{1}$ is the piracy protection parameter for intrinsic features,

$\alpha_{2}$ is the piracy protection parameter for network features,

$\phi\left(\alpha_{2}\right)$ is the fraction of externality supplied by pirates relative to legal users.

If the software is bought, the individual's valuation function is assumed to be the linear sum of the intrinsic valuation for the new software, $A$; the network externality, $B\left[n_{b}(t)+\phi\left(\alpha_{2}\right) n_{p}(t)\right]$; and the disutility from price, $P(t)$. The value of the network externality is determined by the segments of the market, $n_{b}(t)$ and $n_{p}(t)$, that adopt the product. We assume that a pirate adds less value than a legal user by a factor $\phi\left(\alpha_{2}\right)$, which is a decreasing function of the protection on the network features.

As a benchmark to the dynamics that will be introduced subsequently, we consider a static framework. A desirable equilibrium outcome is one where all consumers buy the product. Undesirable equilibrium outcomes are ones where all consumers are either pirating the software or not adopting. Let $(i$, $j$ ) be an ordered pair where $i, j \in\{$ Buy, Pirate, Not Adopt $\}$. The first element in the ordered pair represents the actions of an individual consumer, and the second element represents the state of the remaining population. We want to set conditions on price, $P$, and $\alpha_{1}$ and $\alpha_{2}$ so that (Buy, Buy) is the equilibrium outcome. Let us suppose a piracy-first route is chosen where one induces nonadopters to piracy followed by inducing pirates to buy.

There are three necessary conditions. The first condition is that when no one is adopting the product, the utility from pirating should be greater than the utility from not adopting:

$$
A\left(1-\alpha_{1}\right)>V .
$$

The second condition is that when all are buying the product, the utility from buying should be greater than the utility from pirating:

$$
A+B N-P>A\left(1-\alpha_{1}\right)+B N\left(1-\alpha_{2}\right) .
$$

Implied from this is the third condition that when all are pirating the product, 
the utility from buying is greater than the utility from pirating:

$$
A+B N-P>A\left(1-\alpha_{1}\right)+\phi\left(\alpha_{2}\right) B N\left(1-\alpha_{2}\right) .
$$

Subject to the constraints implied by equations (3)-(5), the firm maximizes its objective function given by equation (1) to obtain the optimal price and protection level.

A parallel analysis can be conducted where nonadopters are shifted to adopting while not allowing piracy. The constraints are that when nobody buys, buying is preferred to not adopting as well as to pirating and that buying is preferred both when all are buying and when all are pirating:

$$
\begin{gathered}
A-V \geq P, \\
P / A-\alpha_{1} \leq 0, \\
P \leq A \alpha_{1}+\phi\left(\alpha_{2}\right) B N \alpha_{2} .
\end{gathered}
$$

The following proposition is obtained (proof in the appendix).

PROPOSITION 1. If $V \leq A$, with a homogenous population,

1. the solution obtained by allowing piracy is more profitable than the solution allowing no piracy,

2. the protection level is higher for the piracy-allowed scenario,

3. the optimal price-protection mix is $\alpha_{2}$ solves $-2 \alpha_{2} \theta_{2}+B N^{2}\left[\alpha_{2} \phi^{\prime}\left(\alpha_{2}\right)\right.$ $+\phi] /(1-\delta)=0$,

$$
\alpha_{1}= \begin{cases}\frac{N A}{2(1-\delta) \theta_{1}} & \text { if } \frac{N A}{2(1-\delta) \theta_{1}} \leq 1-\frac{V}{A} \\ 1-\frac{V}{A} & \text { if } \frac{N A}{2(1-\delta) \theta_{1}}>1-\frac{V}{A},\end{cases}
$$

and $P=A \alpha_{1}+\phi\left(\alpha_{2}\right) B N \alpha_{2}$.

The first result states that some tolerance in piracy protection is always optimal. This is true even when piracy protection is costless, and certainly true when it is costly. Moving to the second result, it may seem surprising that protection is higher for the piracy scenario. However, upon closer examination we see that this is necessary because a higher price can be maintained under this scenario while ensuring that piracy is not the preferred outcome. The profit comparison reveals that the higher price outweighs the cost of higher protection. The third result provides the optimal solution.

We know in practice that the entire market generally does not switch from (Not Adopt) to (Pirate) and then from (Pirate) to (Buy) instantaneously. Rather, there should be a gradual dynamic path that results from market features such as inertia and heterogeneity. This dynamic path is important to model because the profit function depends on the discounted profits over time. 


\section{Dynamic Analysis}

It should be the case that any choice is more likely to occur when it yields relatively higher utility than alternative choices, and less likely when it yields relatively lower utility. Dynamics with this property are known as adaptive dynamics (Milgrom and Roberts 1991).

The general literature on adaptive dynamics can be divided into evolutionary theories and learning theories. Evolutionary theories deal with "survival" of superior actions through higher reproduction rates and "extinction" of inferior actions through lower reproduction rates. Learning theories, on the other hand, deal with players adjusting their beliefs or actions, or both, in response to information on past play. Learning theories, in turn, can be divided into expectation-based models and reinforcement models. Expectation-based models have much to do with players adjusting their beliefs in response to observed past distribution of play, whereas reinforcement models are concerned with players adjusting their propensities over actions in response to their observed past payoffs.

Replicator dynamics is the best known model for evolutionary dynamics, making it a natural choice with which to begin our discussion (Maynard Smith 1982; Hofbauer and Sigmund 1988; Friedman 1991). The model postulates a population of $J$ types, where $J$ is the number of available actions. Let the proportion of type $j$ in period $t$ be denoted by $p_{j}(t)$. Let $\chi(k, j)$ take on the value of one if $k=j$, and zero otherwise. Then, the population distribution at time $t$ is given by

$$
\frac{\dot{p}_{j}(t)}{p_{j}(t)}=\kappa \sum_{k=1}^{J}\left\{\left[\chi(k, j)-p_{k}(t)\right] \sum_{m=1}^{J} U_{k m} p_{m}(t)\right\},
$$

where, $j, k, m \in\{$ Buy, Pirate, Not Adopt $\}$. The left-hand side is the rate of change of type $j$ 's proportion. This is proportional to the difference between the utility experienced by that type over the utility experienced on average, with $\kappa$ being the proportionality constant. Thus, in a population, if type $j$ gets more than average utility, then its proportion increases.

Two concerns may be noted with the direct application of replicator dynamics. First, it assumes that there is no within-type diversity. Second, the underlying biological process of evolution may be inappropriate for describing behavior. Therefore, a dynamic known as standard partial adjustment is described, which is shown to nest the popular replicator dynamics model and is belief-based.

In standard partial adjustment, henceforth referred to as SPA (Cheung and Friedman 1997), a consumer possesses beliefs about the population distribution over choices. A consumer's beliefs about the proportion of others taking action $j$, denoted by $b_{j}(t)$, is updated each period by a weighted average of past and current realizations:

$$
b_{j}(t)=\beta b_{j}(t-1)+(1-\beta) p_{j}(t) .
$$


The parameter $\beta$, which takes a value between zero and one, is the inertia parameter. It captures the effect of availability and quality of information. If $\beta$ is zero, the individual takes a single period to correctly adjust his expected utilities and take a best response action. If $\beta$ is one, the inertia is at its maximum, and the individual stays at the same point forever, never adjusting.

Let $p_{j}(t)$ be the probability that an individual pulled at random from the population will select choice $j$ at time $t$. We now have expected utilities defined as

$$
\begin{aligned}
\mathbf{U}(\mathbf{t}) & =\left(\begin{array}{c}
U_{\text {Buy }}(t) \\
U_{\text {Pirate }}(t) \\
U_{\text {Not Adopt }}(t)
\end{array}\right) \\
& =\left(\begin{array}{c}
A+B\left[N b_{b}(t)+\phi\left(\alpha_{2}\right) N b_{p}(t)\right]-P(t) \\
A\left(1-\alpha_{1}\right)+B\left[N b_{b}(t)+\phi\left(\alpha_{2}\right) N b_{p}(t)\right]\left(1-\alpha_{2}\right) \\
V
\end{array}\right),
\end{aligned}
$$

and

$$
p_{j}(t)=\frac{\exp \left[\lambda U_{j}(t)\right]}{\sum_{k \in J} \exp \left[\lambda U_{k}(t)\right]} .
$$

The parameter $\lambda$ is a scaling parameter also interpreted as the logit noise parameter. As $\lambda$ goes to zero, best replies to beliefs are noisy. As $\lambda$ approaches infinity, best replies become precise. The mapping from expectations to actions is the logit specification.

The logit specification is widely applied in many contexts. The logit specification was made popular by McFadden (1974). By that formulation, for a consumer faced with $J$ alternatives, a random utility model is applied that supposes that disturbances in the utility from each choice are independently and identically distributed with type 1 extreme value distribution. The explanatory variables may be individual characteristics (with coefficients indexed by choice) or choice characteristics. In the individual characteristics case, the logit formulation allows for a rigorous breakdown and characterization of heterogeneity in consumers and its ramification on consumption patterns. For example, Guadagni and Little (1983) incorporate heterogeneity through brand and size loyalty variables. These ideas are taken further in the work of Chintagunta, Jain, and Vilcassim (1991) and Roy, Chintagunta, and Haldar (1996).

In the choice characteristics case, heterogeneity may not be quantified on individual characteristics but is nevertheless accounted for: as long as consumer utility for each choice is approximately normally distributed around a given average utility for that choice, independent from other choices, the probit and its close relative the logit provide a good approximation of the distribution of choice and can be used to assess the impact of choice characteristics on the average utility from each alternative (e.g., Gensch and Recker 1979). The 
problem with this heterogeneity interpretation in the current setting arises due to the repeated nature of choice. That is, the error term in the utility for particular choice by a given consumer in a given time period is assumed independent not only of other consumers' error terms but also of this particular consumer's past error terms. However, to the extent that the "mood shock" interpretation of Sarin and Vahid (1999) is adopted, different consumers have different preferences due to the different moods they wake up with every morning, with these moods independent every morning and not related to individual characteristics. By that interpretation, the logit captures mood heterogeneity without being subject to the above criticism. Other forms of heterogeneity may not be captured without further specification. The discussion of other possible ways to characterize heterogeneity is relegated to the conclusions section (see Sec. VI).

An interpretation that abandons the heterogeneity aspect altogether is the interpretation of the error term as a computation error that is identically and independently distributed for each choice. A close interpretation of the error term attributes it to payoff sensitivity. That is, if two or more choices yield very close payoffs, the consumer would not be likely to invest much effort in evaluating them relative to each other, whereas choices farther apart in payoffs would be more likely to be distinguished, with the highest payoff option chosen with much higher frequency. In the setting at hand, a consumption choice involves a great deal of costly information search regarding product features and assignment of value to various product features relative to alternatives, as well as nonnegligible uncertainty in the process. It is hence not unexpected that some failure to correctly assess one's utility from each choice is to be found. The computation error interpretation is thus appropriate in the setting under investigation. Looking at studies with repeated choice of the nature investigated here, the logit model of noisy best response (McKelvey and Palfrey 1995) has been most prominently used for modeling repeated choice situations. It has also shown remarkable fit in experimental settings. Most prominent in that field are Fudenberg and Levine (1998), Slonim and Roth (1998), Camerer and Ho (1999), Capra and Holt (1999), Erev, BerebyMeyer, and Roth (1999), Stahl (1999), and Sarin and Vahid (2001). In marketing, the use of the logit in a dynamic learning context was pioneered by Chintagunta and Rao (1996).

We have noted that SPA overcomes the limitations of replicator dynamics while nesting its basic form. The following proposition reasserts this, with details relegated to the appendix.

Proposition 2. The SPA nests replicator dynamics (proof in the appendix).

This result is important since it provides another rationale to use SPA, given that replicator dynamics has been shown to capture population behavior in a wide variety of biological and social systems (Maynard Smith 1982). 


\section{A. Illustration}

For illustration, we proceed with the following consumer valuations of $A=\$ 10.76, B N=\$ 4.94$, and $V=\$ 2.74$, taken from a study of online personals services by Haruvy, Mahajan, and Prasad (2000) on a sample of university students. ${ }^{3}$ The questionnaire dealt with self-explicated measures of willingness to pay that can be used to obtain the value of network externality. ${ }^{4}$ Research has shown that simple, self-explicated questionnaires are quite reliable (Srinivasan and Park 1997). The service has an intrinsic value because it is also described as providing features such as e-mail, articles, advice, and restaurant coupons.

For this section, we assume, in line with Takeyama (1994) and Givon et al. (1995), identical protection for intrinsic and externality-related features $\left(\alpha_{1}=\alpha_{2}=\alpha\right)$ and identical externality effects from legal users and pirates $\left(\phi\left(\alpha_{2}\right)=1\right)$ and $\theta=0$. In addition to measurement of customer valuations, additional inputs need to be obtained from managerial judgment. To obtain managerial data on parameters such as the costs of protection and inertia, a standard Delphi technique that pools the information from experts to obtain the best estimate of parameters may be used (Jolson and Rossow 1971). Managers determine how different estimates would affect the outcomes and reevaluate their decisions in the spirit of the decision calculus approach advocated by Little (1970). We assume these are as follows: inertia $\beta$ was set at 0.5 , the center of its $[0,1]$ range, and $\lambda=5$, implying high sensitivity to payoffs. Based on an estimate of a 3-year lifetime for such a service, the time interval, $T$, is set at 36 months. The monthly discount rate, $\delta=0.992$ from the formula $\delta=\left(1+r^{12}\right)^{-1}$ where $r$, the annual industry rate of return on investment, was assumed to be $10 \%$.

For different values of price and protection, profit can be calculated. The maximum profit is achieved at a price of $P^{*}=\$ 10.68$. The protection level of $\alpha^{*}=0.74$ implies that the company should encourage some piracy. This is different from the static case in which the optimal values can be calculated from proposition 1 to be a price of $\$ 11.7$ and protection of 0.75 .

3. As with piracy, online personals have the feature that some consumers pay while others browse for free and are tolerated because they create volume and provide an externality to the paying customers. This second segment faces the same trade-off as the pirates in our modeli.e., to obtain the service for free, they choose to have a lower quality product both in terms of intrinsic utility and network externality since the online personals provider reduces the facilities available to them.

4. The following questions from which $A, B N$, and $V$ may be obtained were asked: (1) What is the maximum amount you would be willing to pay for a monthly subscription if you believed that no one else was presently subscribing to this service? (yields $A-V$ ); (2) What is the maximum amount you would be willing to pay for a monthly subscription if you believed that a majority of attractive Web surfers in search of a significant other are already subscribed to this service? (yields $A+B N-V$ ); and (3) What is the maximum amount you would be willing to pay for a monthly subscription if you believed that a majority of attractive Web surfers in search of a significant other are already subscribed to this service and you believed most of these individuals are not reachable through any other service? (yields $A+B N$ ). 
Following the calculation of the optimal values $P^{*}$ and $\alpha^{*}$, we check the response and robustness of the optimal values to parameter changes. Let $B^{*} \equiv B N$. Comparative statics were conducted on product parameters $A$ and $B^{*}$; the parameters of the dynamic, $\lambda$ and $\beta$; and time parameters, $T$ and $\delta$, by adjusting their values and observing the effects on $\mathrm{P}^{*}$ and $\alpha^{*}$. The values were adjusted from 0.05 to 1.0 for $\beta, 0.1$ to 5.0 for $\lambda, 3$ months to 4 years for $T, 0.64$ to 1.0 for $\delta$, and $\$ 1$ to $\$ 20$ for both $A$ and $B^{*}$.

As might be expected, we find that optimal price is increasing in both $A$ and $B^{*}$. This is because, as the valuation for the product increases, a higher price can be charged. The explanation is straightforward, and the result is robust for all $\left(A, B^{*}\right)$ combinations.

We also find that optimal protection is at maximum for very small $B^{*}$. The reason is that network externality has to be large enough to justify tolerance for piracy. If the network externality is not large enough, tolerance for piracy is never justified. When making the design decision for its software, the firm has a choice of putting in or leaving out externality-enhancing features, such as interactivity between users (e.g., Napster) and chat rooms (e.g., America Online). The implications for the use of piracy in market penetration should be understood. Less intuitive are the following three additional findings:

FINDING 1. Optimal protection should be high if $(a) A+B^{*}$ is only marginally higher than $V,(b) A$ is very large relative to $B^{*}$, or $(c) A$ is very small relative to $B^{*}$.

Explanation. An advantage of tolerating piracy is that price can be high without sacrificing network externalities. For $A+B^{*}$ only marginally higher than $V$, the price that can be charged is low even after tolerating piracy, and hence the benefits from piracy diminish. For example, many shareware providers offer low-value, me-too utilities and also do not protect their software. Not surprisingly, they suffer rather than benefit from piracy (Messitt 2002).

For $A$ large relative to $B^{*}$ there is less value from piracy because it is the intrinsic valuation of the product that is causing people to buy it, and any externality that the pirates may provide is small because of the small value of $B^{*}$ relative to $A$. Products that do not exhibit a great deal of network externality thus should not tolerate piracy. This might explain why the music industry is so opposed to individual piracy as compared to the software industry. In the former case, there are lower network externalities due to the absence of compatibility requirements, and so forth.

Part $c$ of the finding is explained by the fact that once the market had moved to pirating the product, it will be difficult to wean it toward buying the product if the intrinsic value and protection are low. An example is the Internet personals service that has mainly network externality value and less intrinsic value. According to this result, nonregistered members should not be provided with much utility.

FINDING 2. Optimal price is increasing in both discount factor, $\delta$, and the number of periods, $T$. For $\delta$, unreasonably low or for a very small number 
of periods, optimal protection is at the maximum, and optimal price is low. For reasonable $\delta$ and a number of periods over 6 months, optimal protection is not very sensitive to time variables.

Finding 3. As long as the noise parameter, $\lambda$, does not approach zero, and the inertia parameter, $\beta$, does not approach one-in other words, as long as consumers are somewhat sensitive to the surplus, and inertia does not keep them in place-the resulting optimal price and protection are fairly flat in $\lambda$ and $\beta$, implying that the recommendations are robust. The exceptions to this flatness are as follows:

a) As $\beta$ approaches one (perfect inertia), optimal protection approaches one, and for larger values of $\lambda$, price drops sharply. When $\beta$ approaches one, movement from piracy to buying would occur very slowly (or not at all when $\beta$ is exactly one), and since the firm discounts the very distant future, a piracy-first strategy is not profitable. Hence, protection should be high and price low.

Explanation. For $\delta$ values roughly below 0.9 (corresponding to yearly returns of over $130 \%$ ), the discounting of future periods is too large for future profits to be a real consideration. Hence, immediate short-term profits are a focus, and an indirect piracy-first approach is unprofitable. Similarly, if the product is short-lived, the appropriate focus in on immediate profit, and a piracy-first approach is not so lucrative. When short-term profit is the focus, protection needs to be at or near maximum and price at or near the outside valuation. When long-term profit is the focus (many time periods and high $\delta)$, protection should be low and prices high.

b) As $\lambda$ approaches zero, optimal protection approaches one, and price rises sharply. This is because, due to noise, there are a significant number of customers who have a high valuation for the product regardless of the network size. Hence, price can be high and consumers will still buy. A piracy-first strategy, which is intended to ultimately raise the price, is not profitable.

The phase diagram depicts how the adoption process will evolve over time and the equilibrium to which it will converge, leading to additional insights. The phase diagram in figure 1 is developed using parameter values as described above.

Two conclusions may be drawn from this diagram: First, it is seen that the proportion of pirates rises and then falls, while the proportion of buyers rises and then remains stable. As the proportion of buyers rises, so does profit. Second, management should expect an initial period of little or no profit since the group of adopters is mainly composed of pirates. However, due to the decay of profit over time, resulting from discounting, this initial period cannot be too long. 


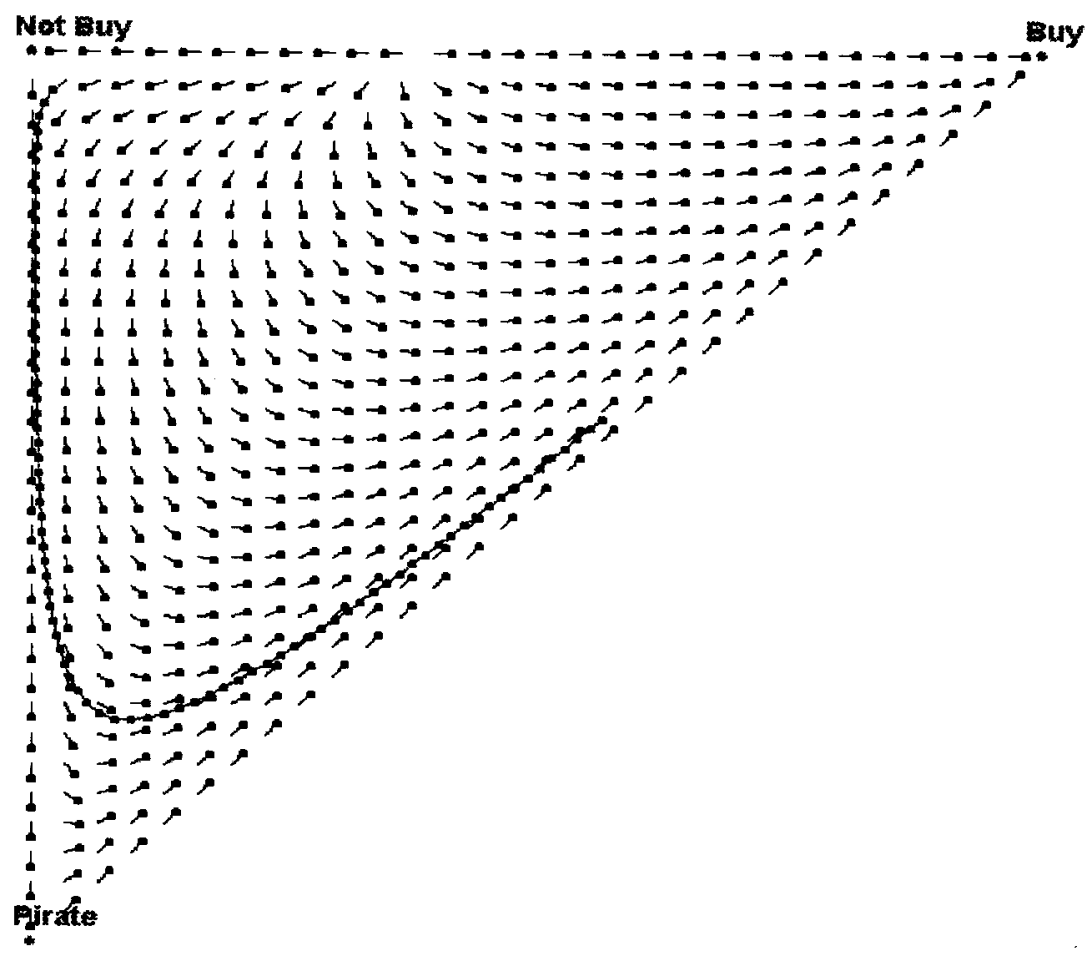

FIG. 1.-Phase diagram for adoption process

\section{B. Dynamic Pricing Scenario}

The preceding analysis was conducted with static price and protection controls. Static protection controls are appropriate, given that protection is a product design decision that cannot be rapidly adjusted during the short product life cycle of most software. A static price is appropriate when the firm cannot quickly change prices due to short product life cycles and marketing compulsions, such as pricing being a retailer decision when the software is sold through intermediaries, competitive pricing, and consumer skepticism and annoyance at rapid price changes.

Though static controls have been used, there is no inherent limitation in the methodology that prevents the calculation of optimal dynamic controls. Continuing with the parameter values from the previous illustration, we examine the effect of being able to change the software's price once a month for 12 months on the price-protection decision.

Recall that with static prices the optimal values were $P^{*}=\$ 10.68$ and $\alpha^{*}=0.74$. The result of the dynamic pricing analysis is that the optimal protection level is now $\alpha^{*}=0.93$. The optimal price path is shown in figure 2 . 


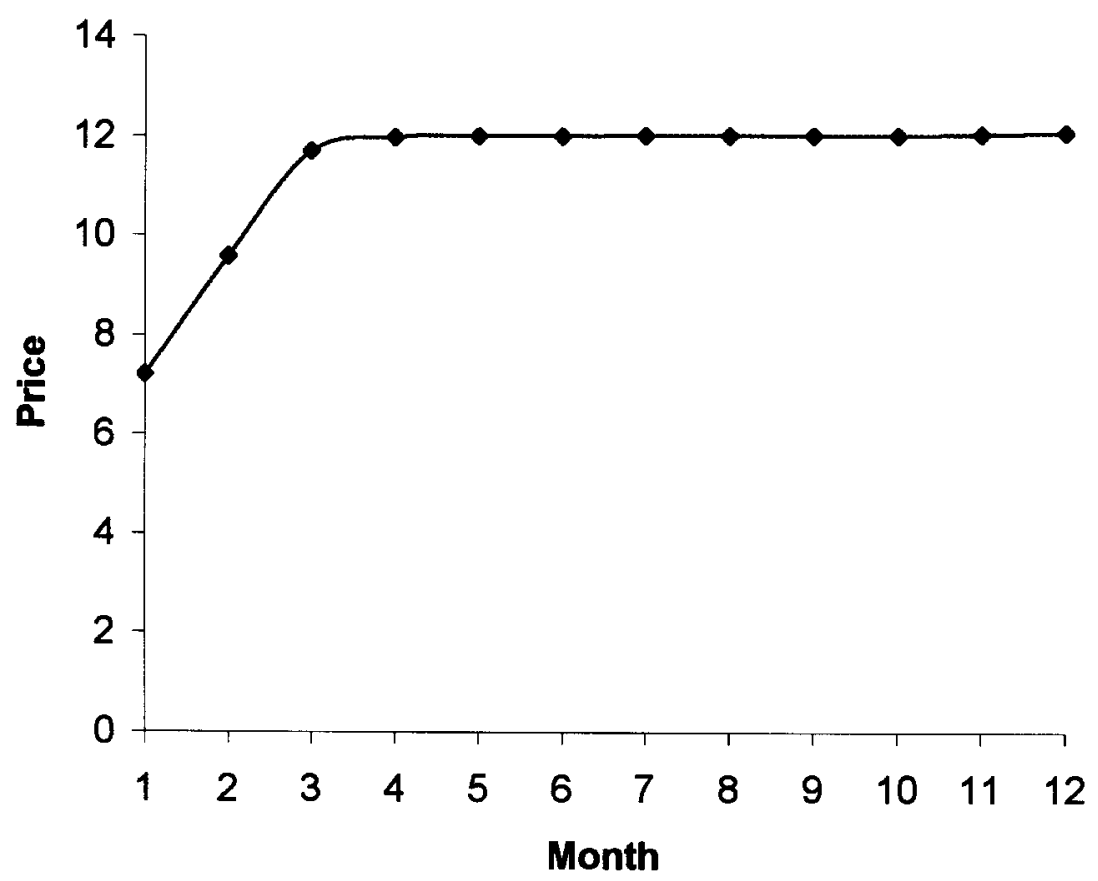

FIG. 2.-Optimal price path

Upon examining this graph (and also adjusting the parameter values and examining additional graphs), we observe a penetration pricing strategy. Note also that the price path starts at a lower value than the optimal price of $\$ 10.68$ obtained with a static price analysis. However, over the long run, the price rises above the static price solution. This again implies that the initial price should be kept low to build up a network if price can later be increased to take advantage of a larger network.

Finally, the protection level when the price is dynamic is higher than in the case with static price. This is because a low price, particularly in the beginning, supplements the role of piracy in encouraging early adoption. However, as the value $\alpha^{*}=0.93$ indicates, it does not completely displace the use of piracy. In conclusion, it is clear that the pricing strategy of the software should be evaluated together with its protection strategy to yield the best profit to the firm.

\section{Extension to Nonsubscription Software}

If the software is not subscription based, a few modifications to the proposed model are required to model customer adoption. Unlike in the subscription setup, here a buyer cannot switch to piracy or nonuse following the purchase 
of the product. Similarly, in accordance with the treatment by Givon et al. (1995) of software as a durable good, a pirate cannot unadopt the product or switch to buying. Only the nonuser faces the three choices of purchase, piracy, and not adopting that the entire population was facing initially.

The consumer in the present dynamic model updates his or her expectations as in equation (7). The firm's objective function remains essentially the same:

$$
\max _{\theta_{1}, \theta_{2}, P(1), P(2), \ldots, P(T)}-\theta_{1} \alpha_{1}^{2}-\theta_{2} \alpha_{2}^{2}+\sum_{t=0}^{T} \delta^{t} P(t) \nu_{b}(t) .
$$

Note that $n_{b}(t)$ from equation (1) was replaced by $\nu_{b}(t)$. Whereas $n_{b}(t)$ denoted the number of legal users of the software, $\nu_{b}(t)$ denotes only firsttime purchasers, or "switchers," from nonuse. This is because, in the present framework, a legal user has no need to make another purchase and hence no longer enters the profit stream.

Recalling that $n_{n}(t-1)$ is the number of nonusers at time $t-1$, we have:

$$
\nu_{b}(t)=n_{n}(t-1) p_{b}(t),
$$

where $p_{b}(t)$ is the probability of a nonuser buying at time $t$. Another important notation is $\nu_{p}(t)$, the number of nonusers switching to pirate at time $t$. The $n$ 's are updated as follows:

$$
\begin{aligned}
& n_{b}(t)=n_{b}(t-1)+\nu_{b}(t), \\
& n_{p}(t)=n_{p}(t-1)+\nu_{p}(t), \\
& n_{n}(t)=N-n_{b}(t)-n_{p}(t) .
\end{aligned}
$$

Past buyers and pirates in periods prior to $t$, given that the software is durable, have zero probability to buy in period $t$. The probabilities of purchase for nonusers are determined as before, through the logit function.

The expectations to be inserted in the logit are updated based on expected utilities from each choice, where such expectations are updated with inertia as before. The vector below, superscripted by $n$ to indicate nonusers, denotes the utility vector for a nonuser at time $t$ :

$$
\left(\begin{array}{c}
U_{\text {Buy }}^{n}(t) \\
U_{\text {Pirate }}^{n}(t) \\
U_{\text {Not Adopt }}^{n}(t)
\end{array}\right)=\left(\begin{array}{c}
A+B\left[n_{b}(t)+\phi\left(\alpha_{2}\right) n_{p}(t)\right]-P(t) \\
A\left(1-\alpha_{1}\right)+B\left[n_{b}(t)+\phi\left(\alpha_{2}\right) n_{p}(t)\right]\left(1-\alpha_{2}\right) \\
V
\end{array}\right) .
$$

For illustration we use the empirical data used by Givon et al. (1995, 1997) on 1987-92 word processing, spreadsheet, and personal computers sales data. The analysis closely follows that found in Givon et al. (1995) to aid comparison. The data are at the category level and, therefore, the price used is the closest average price of $\$ 395$ for both word processors and spreadsheets 
that could be verified for that period (International Data Corporation 1988a, 1988b).

The parameter values selected are those that minimized the sum of squared deviations between the simulated number of legal purchases and the actual number of legal purchases, over periods for which data were available. The process is to repeatedly guess the parameter values and simulate the number of legal purchases by using equations (7) and (9)-(15). The number of pirates in the simulation is used only to obtain the simulated number of legal buyers, since data on piracy are not available to compare against. This approach is advocated by Givon et al. (1995), who noted that direct data on piracy would be exceedingly difficult to obtain.

For estimation, the simulated annealing technique was used (Kirkpatrick, Gelatt, and Vecchi 1983; Fox 1988; Otten 1989). This algorithm stochastically simulates the slow cooling of a physical system: it iteratively proposes changes and either accepts or rejects each change, depending on the "temperature." When the temperature is high, all changes are accepted, and we simply move at random, ignoring the objective function. When the temperature is zero, changes are accepted only if the objective function decreases, an algorithm also known as hill climbing. The algorithm proposes many changes, starting at a high temperature and exploring the state space, and gradually decreasing the temperature to zero. It has been shown that if the temperature decreases sufficiently slowly, the probability of being in a global optimum tends to certainty (Hajek 1988).

For the estimation results shown below, as in the subscription case, we assumed $\alpha_{1}=\alpha_{2}=\alpha$ and identical externality effects from legal users and pirates, $\phi\left(\alpha_{2}\right)=1$. Alternative assumptions were tested but resulted in no statistically significant improvement. ${ }^{5}$ The actual and estimated sales for each category are shown in figure 3.

The following parameter estimates were obtained (see table 1). The $R^{2}$ goodness-of-fit measures are 0.517 and 0.749 for word processors and spreadsheets, respectively, slightly lower than the reported values of 0.563 and 0.789 from Givon et al. (1995). Also similar to that paper, we find that the proportion of pirates is extremely high, for example, approximately six of every seven adopters of word processors had a pirated copy. However, some unique additional insights may be obtained.

The intrinsic valuation, $A$, and the network effects, $B^{*}$, for both word processors and spreadsheets is high. In particular, the intrinsic valuation is higher than the value from the best outside option in both cases and, for word processors, from the average price. Since the intrinsic valuation for spreadsheets is lower than the average price, market penetration would have been much slower in the absence of piracy and network externalities.

5. Specifically, we allowed $\alpha_{1}$ and $\alpha_{2}$ to be distinct and tested the alternative specification $\phi\left(\alpha_{2}\right)=1-\alpha_{2}$. In both cases, no improvement was found to justify the more complex formulations. 


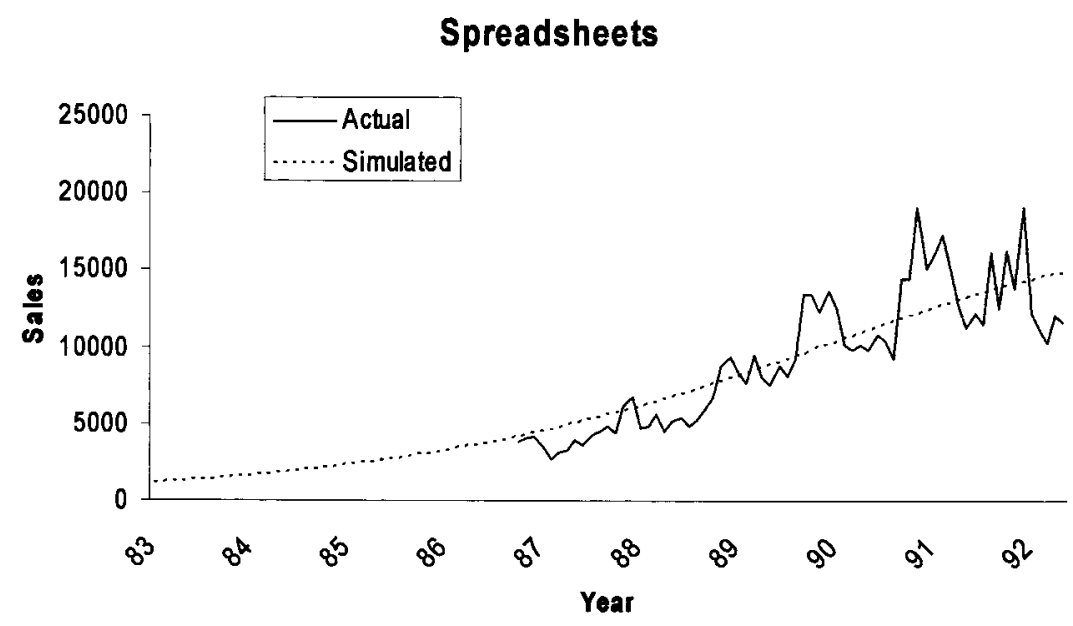

Wordprocessors

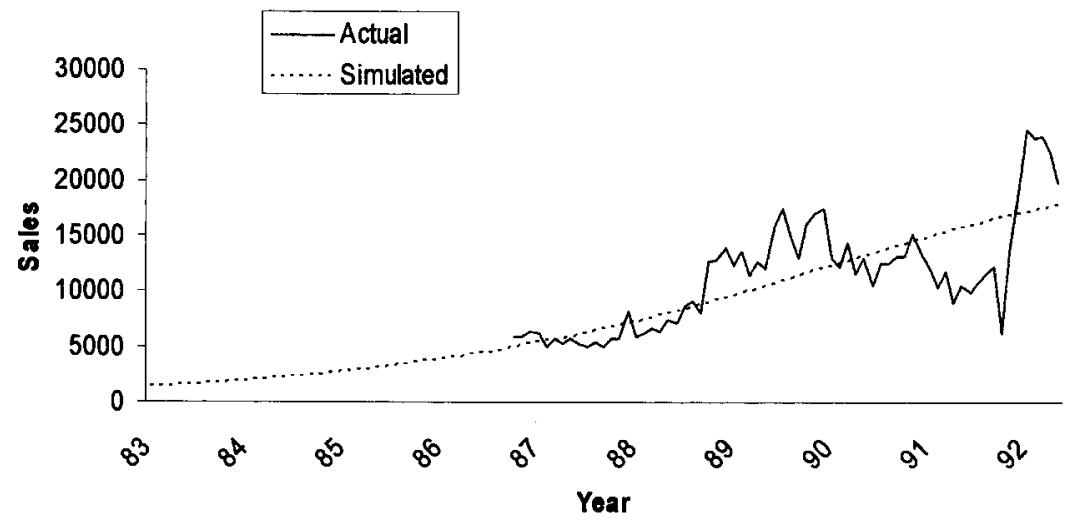

FIG. 3.-Actual and estimated legal sales of spreadsheets and word processors in the United Kingdom.

It is interesting to note that the inertia parameter is close to one, indicating that despite the advantage of adopting these products, market penetration was slow. To reduce inertia, a marketing campaign advertising the growing usage and network benefits of the software would have been helpful. Finally, the value of $\lambda$ is low enough that a few nonadopters will be seen in equilibrium yet high enough that people are sensitive to payoff differences, and most of the population will end up adopting the software due to the lower utility from the outside option.

We can compute whether firm behavior was optimal given the parameter estimates. If cost of protection is ignored, since information on it is lacking, it turns out that the optimal piracy protection for both categories is full pro- 


\begin{tabular}{lcc} 
TABLE 1 & \multicolumn{2}{c}{ Parameter Estimates } \\
\hline & Word Processors & Spreadsheets \\
\hline$\alpha$ & .41 & .30 \\
$\beta$ & .99 & .99 \\
$\lambda$ & .16 & .13 \\
$A(\$)$ & 450 & 296 \\
$B^{*}(\$)$ & 593 & 257 \\
$V(\$)$ & 119 & 79 \\
\hline \multicolumn{2}{l}{ NotE. - Let $B^{*}$ denote the additional valuation if the entire pop- } \\
ulation adopted.
\end{tabular}

tection. This recommendation is made with the caveat that the cost of protection may have been significant at the time.

\section{Conclusions}

Any software firm would like to see the market adopt its product. However, due to network externalities, new software may be unattractive to customers until a large number of other customers have already purchased the product and established a user base, or network. As a consequence, the customers may all remain lodged in the initial state of nonadoption, and the product will fail. In such a situation, it may be possible to first shift some individuals from nonadoption to piracy and to use piracy strategically to establish the initial network. When the network size increases, if network externalities are present, the utility from the product would rise, and the number of buyers would progressively rise as well.

This article advances previous research on piracy to show how price and protection may be optimally managed. Piracy tolerance is viable only if projected gains from such a strategy are more than substantial. When piracy control is costly, information is precise, market penetration is rapid, externalities are low, future profits are greatly discounted, customer inertia is low, or product life is short, tolerance of piracy is not recommended.

The analysis characterized adoption dynamics due to piracy and buying and the resulting profit stream over time. An expression for the discounted profit stream was obtained and maximized to yield the best price and protection decisions. Such an approach allows for the incorporation and joint optimization of the critical firm decision variables of price and protection. The dynamic analysis extends the previously static piracy literature on network externalities to a dynamic framework. We use dynamics emerging from adaptive agents learning over time and show this to be consistent with the evolutionary game theoretical framework.

The objective was to help managers achieve a profitable launch of new software by modeling the interplay of factors that enhance or deter the ability of the product to achieve a full and fast market penetration. The model showed a good fit of the adoption pattern of software. It can be used to provide normative guidelines to managers in software firms to determine the price of 
the product and the level of software protection. Inputs to the model could be obtained prior to the launch of the software and need not include data on past piracy behavior that are difficult to obtain and fraught with bias. Hence, it is easily applicable.

Several avenues for future research may be noted. From a substantive perspective, software marketers sometimes offer consumers freeware that can also provide network externalities. A model can be developed that gives the alternatives of $\{$ Buy, Freeware, Pirate, Not Adopt $\}$ to the customer. Extension to competitive markets where customers have even more alternatives should also be investigated to see if piracy plays any useful role in such markets.

From a methodological perspective, a limitation of the model is that the role of an individual's past actions on current behavior has not been incorporated. Thus, due to familiarity with usage, a pirate or a buyer in the last period may be more likely to be an adopter in the next period. Note that including such an effect could actually increase the usefulness of piracy. Another limitation in the extension to model durable software is that the role of future price expectations on consumer demand has not been captured (e.g., Levinthal and Purohit 1989). Finally, the discussion of heterogeneity in Section IV was limited to nonsystematic shocks. To the extent that populations with systematically divergent patterns of behavior may be identified, the error term $\lambda$ is insufficient to account for such differences. Subpopulations such as corporate adopters and individual adopters could be incorporated into the model if these were believed to have distinct behaviors and if data were available to empirically characterize these differences. It is also known that propensities to pirate differ geopolitically. Extending the model along these lines deserves consideration.

\section{Appendix}

\section{Proof of Propositions}

\section{Proof of Proposition 1}

To move the market to the situation where everyone buys the product, it is necessary that when the market has nobody adopting, not adopting should not be individually optimal. We examine two routes to accomplish this, $(a)$ first, by making piracy preferred to not adopting and later ensuring that piracy is worse than buying after a significant number of adopters have been generated, and $(b)$ by making buying preferred to no adoption at the outset. We examine the first route first.

\section{A. The Piracy-First Route}

The requirement is that piracy is preferred to not adopting when nobody is adopting, that is,

$$
A\left(1-\alpha_{1}\right) \geq V
$$




$$
\Rightarrow \alpha_{1} \leq 1-\text { VIA }
$$

If indifferent, we assume piracy is the default action. The second condition is that when everyone is pirating, the utility from buying is greater than the utility from pirating. This also implies that when all are buying, buying is preferred to pirating. We get:

$$
\begin{gathered}
A+\phi\left(\alpha_{2}\right) B N-P \geq A\left(1-\alpha_{1}\right)+\phi\left(\alpha_{2}\right) B N\left(1-\alpha_{2}\right) \\
\Rightarrow P \leq A \alpha_{1}+\phi\left(\alpha_{2}\right) B N \alpha_{2} .
\end{gathered}
$$

If indifferent, we assume buying is the default action. The firm maximizes its objective function subject to the constraints in equations (A2) and (A4).

$$
\max _{\theta_{1}, \theta_{2, P}}-\theta_{1} \alpha_{1}^{2}-\theta_{2} \alpha_{2}^{2}+\sum_{t=0}^{T} \delta^{t} P n_{b t},
$$

subject to equations (A2) and (A4).

To solve this inequality constrained problem form the Lagrangian, L, with Lagrange multipliers $\lambda_{1}$ and $\lambda_{2}$ for equations (A2) and (A4), respectively:

$$
\begin{aligned}
L= & -\theta_{1} \alpha_{1}^{2}-\theta_{2} \alpha_{2}^{2}+\frac{P N}{1-\delta}-\lambda_{1}\left(\alpha_{1}-1+V / A\right) \\
& -\lambda_{2}\left[P-A \alpha_{1}-B N \alpha_{2} \phi\left(\alpha_{2}\right)\right] .
\end{aligned}
$$

The necessary conditions are:

a) $N /(1-\delta)-\lambda_{2}=0$,

b) $-2 \alpha_{1} \theta_{1}-\lambda_{1}+A \lambda_{2}=0$,

c) $-2 \alpha_{2} \theta_{2}+\lambda_{2} B N\left[\alpha_{2} \phi^{\prime}\left(\alpha_{2}\right)+\phi\right]=0$,

d) $\lambda_{1}\left(\alpha_{1}-1+V / A\right)=0$

e) $\lambda_{2}\left[P-A \alpha_{1}-\phi\left(\alpha_{2}\right) B N \alpha_{2}\right]=0$,

f) $\alpha_{1}-1+V / A \leq 0$,

g) $P-A \alpha_{1}-\phi\left(\alpha_{2}\right) B N \alpha_{2} \leq 0$,

h) $\lambda_{1} \geq 0, \lambda_{2} \geq 0$.

From condition $a$, we get $\lambda_{2}=N /(1-\delta)$. Since this value is greater than zero, therefore the constraint in equation (A4) binds. We have

$$
P=A \alpha_{1}+\phi\left(\alpha_{2}\right) B N \alpha_{2}
$$

Inserting the value $\lambda_{2}=N /(1-\delta)$ into condition $c$, we obtain the implicit solution for $\alpha_{2}$.

$$
-2 \alpha_{2} \theta_{2}+B N^{2}\left[\alpha_{2} \phi^{\prime}\left(\alpha_{2}\right)+\phi\right] /(1-\delta)=0 .
$$

Inserting the value $\lambda_{2}=N /(1-\delta)$ into condition $b$, we obtain the following two cases for $\alpha_{1}$ (corresponding to $\lambda_{1}=0$ and $\lambda_{1}>0$ ). 
Case 1: If

$$
\begin{aligned}
\frac{N A}{2(1-\delta) \theta_{1}} & \leq 1-\frac{V}{A}, \\
\alpha_{1} & =\frac{N A}{2(1-\delta) \theta_{1}},
\end{aligned}
$$

and inserting from equations (A8) and (A9) into equation (A7) would give the price.

Case 2: If

$$
\begin{array}{r}
\frac{N A}{2(1-\delta) \theta_{1}}>1-\frac{V}{A}, \\
\alpha_{1}=1-\frac{V}{A},
\end{array}
$$

and inserting from equations (A8) and (A10) into equation (A7) would give the price.

\section{B. The No-Piracy Route}

There are two requirements: the first is a pair of conditions that when nobody buys, buying is preferred to not adopting as well as to pirating:

$$
\begin{gathered}
A-V \geq P, \\
P / A-\alpha_{1} \leq 0 .
\end{gathered}
$$

The other condition, that buying is preferred both when all are buying and when all are pirating, must also be met:

$$
P \leq A \alpha_{1}+\phi\left(\alpha_{2}\right) B N \alpha_{2} .
$$

But equation (A13) is implied by equation (A12) and can be ignored. The Lagrangian is

$$
L=-\theta \alpha_{1}^{2}-\theta_{2} \alpha_{2}^{2}+\frac{P N}{1-\delta}-\lambda_{1}(P+V-A)-\lambda_{2}\left(P / A-\alpha_{1}\right)
$$

The necessary conditions are:

i) $N /(1-\delta)-\lambda_{1}-\lambda_{2} / A=0$,

j) $-2 \alpha_{1} \theta_{1}-\lambda_{1}+\lambda_{2}=0$,

k) $-2 \alpha_{2} \theta_{2}=0$,

l) $\lambda_{1}(P+V-A)=0$,

m) $\lambda_{2}\left(P / A-\alpha_{1}\right)=0$,

n) $P+V-A \leq 0$,

o) $P / A-\alpha_{1} \leq 0$,

p) $\lambda_{1} \geq 0, \lambda_{2} \geq 0$.

From condition $k$ we obtain

$$
\alpha_{2}=0
$$


From conditions $i$ and $j$ we get

$$
\lambda_{2}=\frac{N /(1-\delta)+2 \alpha_{1} \theta_{1}}{(1+1 / A)}>0
$$

Hence condition $o$ is binding, that is, $\alpha_{1}=P / A$, and

$$
\lambda_{1}=\frac{N /(1-\delta)-2 \alpha_{1} \theta_{1} / A}{(1+1 / A)} .
$$

There are two cases corresponding to $\lambda_{1}=0$ and $\lambda_{1}>0$, respectively.

Case 1: If

$$
\begin{gathered}
\frac{N A}{2(1-\delta) \theta_{1}} \leq 1-\frac{V}{A}, \\
\alpha_{1}=\frac{N A}{2(1-\delta) \theta_{1}}, \text { and } P=\frac{A^{2} N}{2 \theta_{1}(1-\delta)} .
\end{gathered}
$$

Case 2: If

$$
\begin{gathered}
\frac{N A}{2(1-\delta) \theta_{1}}>1-\frac{V}{A}, \\
\alpha_{1}=1-\frac{V}{A}, \text { and } P=A-V .
\end{gathered}
$$

\section{Comparison of the Piracy-First Route and the No-Piracy Route}

In all cases the profit expression is $\Pi=-\theta_{1} \alpha_{1}^{2}-\theta_{2} \alpha_{2}^{2}+[P N /(1-\delta)]$, and we can compare the profit under the two different cases, $\left[N A / 2(1-\delta) \theta_{1}\right]>1-V / A$ and $\left[N A / 2(1-\delta) \theta_{1}\right] \leq 1-V / A$, by inserting the values of $P, \alpha_{1}$, and $\alpha_{2}$. Inserting the values of $P$ and $\alpha_{1}$ corresponding to these two cases, it will be seen that the profit in the piracy-first route is greater in both cases if the following condition is met:

$$
B N^{2} \phi\left(\alpha_{2}\right) /(1-\delta)-\theta_{2} \alpha_{2}>0
$$

subject to equation (A8).

Substituting $\alpha_{2} \theta_{2}=B N^{2}\left[\alpha_{2} \phi^{\prime}\left(\alpha_{2}\right)+\phi\right] / 2(1-\delta)$ from conditions shown in equation (A8) into equation (A18) allows us to write it as

$$
\phi-\alpha_{2} \phi^{\prime}\left(\alpha_{2}\right)>0 \text {. }
$$

This is clearly true since $\phi^{\prime}\left(\alpha_{2}\right)<0$. Thus, the piracy-first approach always does better than the no-piracy approach. Q.E.D. 


\section{Proof of Proposition 2: Relationship between SPA and Replicator Dynamics}

Define $w_{j}(t)=\lambda U_{j}(t)$. The change in $p_{j}(t)$ of equation (9) can be calculated as follows:

$$
\dot{p}_{j}(t)=\sum_{k} \frac{\partial p_{j}(t)}{\partial w_{j}(t)} \dot{w}_{k}(t)
$$

Note that $\dot{w}_{j}(t)$ can be approximated by

$$
\dot{w}_{j}(t) \approx w_{j}(t+1)-w_{j}(t)=(1-\beta)\left[\lambda U_{j} p_{j}(t)-w_{j}(t)\right] .
$$

Plugging equation (A21) into equation (A20) and some algebra results in

$$
\frac{\dot{p}_{j}(t)}{p_{j}(t)}=(1-\beta)\left\{\lambda\left(g_{t j}-\bar{g}_{t}\right)-\left[\ln p_{j}(t)-\sum_{k} p_{k}(t) \ln p_{k}(t)\right]\right\},
$$

where $g_{t j}=\sum_{m=1}^{J} U_{j m} p_{m}(t)$, and $\bar{g}_{t}=p_{k}(t) \sum_{m=1}^{J} U_{k m} p_{m}(t)$. Compare this to the standard replicator dynamics of equation (6), shown below:

$$
\frac{\dot{p}_{j}(t)}{p_{j}(t)}=\kappa \sum_{k=1}^{J}\left[\left[\chi(k, j)-p_{k}(t)\right] \sum_{m=1}^{J} U_{k m} p_{m}(t)\right] .
$$

And one sees that the replicator model is nested within equation (A22). That is, when $\lambda=(\kappa / 1-\beta)$, and $\beta \rightarrow 1$, the model of equation (A22) converges to equation (A23). Q.E.D.

\section{References}

Baily, Jeff, and Scott Kilman. 1998. Taking credit: Here's what's driving some lenders crazy: Borrowers who think. Wall Street Journal (February 20), A1.

Bevan, Kate. 2001. Anti-piracy feature sparks controversy: XP activation debate. Financial Times, October 17, 10.

Business Software Alliance (BSA). 2000. BSA bids against online auction software pirates. BSA Press Room. http://www.bsa.org/uk/press/newsreleases/2000-11-14.423.phtml.

2002. Seventh annual BSA global piracy study 2002. http://www.bsa.org.

Camerer, Colin F., and Teck H. Ho. 1999. Experience weighted attraction learning in normal form games. Econometrica 67:827-74.

Capra, Monica, and Charles A. Holt. 1999. Anomalous behavior in a traveler's dilemma? American Economic Review 89:678-90.

Chatterjee, Rabikar, and Jehoshua Eliashberg. 1990. The innovation diffusion process in a heterogeneous population: A microeconomic approach. Management Science 19:187-200.

Chen, Yongmin. 1997. Paying customers to switch. Journal of Economics and Management Strategy 6:877-97.

Cheung, Yin-Wong, and Daniel Friedman. 1997. Individual learning in normal form games: Some laboratory results. Games and Economic Behavior 19:46-79.

Chintagunta, Pradeep K., Dipak C. Jain, and Naufel J. Vilcassim. 1991. Investigating heterogeneity in brand preferences in logit models for panel data. Journal of Marketing Research 28:417-29.

Chintagunta, Pradeep K., and Vithala R. Rao. 1996. Pricing strategies in a dynamic duopoly: A differential game model. Management Science 42:1501-14.

Conner, Kathleen R., and Richard P. Rumelt. 1991. Software piracy: An analysis of protection strategies. Management Science 37:125-39.

Crawford, Vincent P. 1991. An evolutionary interpretation of Van Huyck, Battalio and Beil's experimental results on coordination. Games and Economic Behavior 3:25-59.

. 1995. Adaptive dynamics in coordination games. Econometrica 63:103-44. 
Dhebar, Anirudh, and Shmuel S. Oren. 1985. Dynamic aspects of network access pricing. Marketing Science 4:336-51.

Erev, Ido, Yoella Bereby-Meyer, and Al Roth. 1999. The effect of adding a constant to all payoffs: Experimental investigation, and implications for reinforcement learning models. Journal of Economic Behavior and Organization 39:111-28.

Farrell, Joseph, and Garth Saloner. 1986. Installed base and compatibility: Innovation, product preannouncements, and predation. American Economic Review 76:940-55.

Fox, Geoffrey C. 1988. The hypercube and the Caltech concurrent computation program: A microcosm of parallel computing. In Special purpose computers, ed. B. J. Alder. Boston: Academic Press.

Friedman, Daniel. 1991. Evolutionary games in economics. Econometrica 59:637-66.

Fudenberg, Drew, and David K. Levine. 1998. The theory of learning in games. Cambridge, MA: MIT Press.

Fudenberg, Drew, and Jean Tirole. 2000. Customer poaching and brand switching. Rand Journal of Economics 31:634-57.

Gensch, Dennis H., and Wilfried W. Recker. 1979. The multinomial, multiattribute choice model. Journal of Marketing Research 16:124-32.

Givon, Moshe, Vijay Mahajan, and Eitan Muller. 1995. Software piracy: Estimation of lost sales and the impact on software diffusion. Journal of Marketing 59:29-37.

- 1997. Assessing the relationship between the user-based market share and unit salesbased market share for pirated software brands in competitive markets. Technological Forecasting and Social Change 55:131-44.

Goeree, Jacob K., and Charles A. Holt. 1999. Stochastic game theory: For playing games, not just for doing theory. Proceedings of the National Academy of Sciences 96:10564-67.

Guadagni, Peter M., and John D. C. Little. 1983. A logit model of brand choice calibrated on scanner data. Marketing Science 2:203-39.

Gurnami, Haresh, and Kamalakar Karlapalem. 2001. Optimal pricing strategies for internet-based software dissemination. Journal of the Operational Research Society 52:64-70.

Hajek, Bruce. 1988. Cooling schedules for optimal annealing. Mathematics of Operational Research 13:311-29.

Haruvy, Ernan, Vijay Mahajan, and Ashutosh Prasad. 2000. Measuring network effects in online services. Working paper, University of Texas at Dallas, School of Management.

Hofbauer, Josef, and Karl Sigmund. 1988. The theory of evolution and dynamical systems. Cambridge, MA: Cambridge University Press.

International Data Corporation. 1988a. Spreadsheet software. Framingham, MA: International Data Corporation.

1988b. Word processing software. Framingham, MA: International Data Corporation.

Jain, Dipak, Vijay Mahajan, and Eitan Muller. 1995. An approach for determining optimal product sampling for the diffusion of a new product. Journal of Product Innovation Management 12: 124-35.

Jensen, Richard. 1982. Adoption and diffusion of an innovation of uncertain profitability. Journal of Economic Theory 27:182-93.

Jolson, Marvin A., and Gerald L. Rossow. 1971. The Delphi process in marketing decision making. Journal of Marketing Research 8:443-48.

Katz, Michael, and Carl Shapiro. 1985. Network externalities, competition and compatibility. American Economic Review 75:424-40.

Keller, John J. 1997. Telecommunications: Best phone discounts go to hardest bargainers. Wall Street Journal, February 13, B1.

Kirkpatrick, Scott, Charles D. Gelatt, and Mario P. Vecchi. 1983. Optimization by simulated annealing. Science 220 (May): 671-80.

Lattin, James M., and John H. Roberts. 1989. Modeling the role of risk-adjusted utility in the diffusion of innovations. Working Paper no. 1019, Stanford University, Graduate School of Business.

Levinthal, Daniel, and Devevrat Purohit. 1989. Durable goods and product obsolescence. Marketing Science 8:35-57.

Little, John D. C. 1970. Models and managers: The concept of a decision calculus. Management Science 16:466-85.

Maynard Smith, John. 1982. Evolution and the theory of games. Cambridge: Cambridge University Press. 
McFadden, Daniel. 1974. Conditional logit analysis of qualitative choice behavior. In Frontiers in econometrics, ed. P. Zerembka. New York: Academic Press.

McKelvey, Richard D., and Thomas R. Palfrey. 1995. Quantal response equilibria for normal form games. Games and Economic Behavior 10:6-38.

Messitt, Colin. 2002. Why do people register, does crippling work, does anybody really know? Available at http://hackvan.com/pub/stig/articles/why-do-people-register-shareware.html.

Milgrom, Paul, and John Roberts. 1991. Adaptive and sophisticated learning in normal-form games. Games and Economic Behavior 3:82-100.

Nascimento, Fernando, and Wilfried R. Vanhonacker. 1988. Optimal strategic pricing of reproducible consumer goods. Management Science 34 (August): 921-37.

Oren, Shmuel S., and R. G. Schwartz. 1988. Diffusion of new products in risk sensitive markets. Journal of Forecasting 7:273-87.

Otten, Ralph H. J. M., and Lukas P. P. P. van Ginneken. 1989. The annealing algorithm. Boston: Kluwer Academic.

Padmanabhan, V., Surendra Rajiv, and Kannan Srinivasan. 1997. New products, upgrades and new releases: A rationale for sequential product introductions. Journal of Marketing Research 34:456-72.

Prasad, Ashutosh, and Vijay Mahajan. 2003. How many pirates should a software firm tolerate? An analysis of piracy protection on the diffusion of software. International Journal of Research in Marketing 20:337-53.

Roy, Rishin, Pradeep K. Chintagunta, and Sudeep Haldar. 1996. A framework for investigating habits, "the hand of the past," and heterogeneity in dynamic brand choice. Marketing Science 15:280-99.

Sarin, Rajiv, and Farshid Vahid. 1999. Payoff assessments without probabilities: A simple dynamic model of choice. Games and Economic Behavior 28:294-309.

. 2001. Predicting how people play games: A simple dynamic model of choice. Games and Economic Behavior 34:104-22.

Slonim, Robert, and Al Roth. 1998. Learning in high stakes ultimatum games: An experiment in the Slovak Republic. Econometrica 66:569-96.

Srinivasan, V., and Chan S. Park. 1997. Surprising robustness of the self-explicated approach to customer preference structure measurement. Journal of Marketing Research 34:286-91.

Stahl, Dale O. 1999. Evidence based rule learning in symmetric normal-form games. International Journal of Game Theory 28:111-30.

Stoneman, Paul. 1981. Intra-firm diffusion, Bayesian learning and profitability. Economic Journal 91:375-88

Takeyama, Lisa N. 1994. The welfare implications of unauthorized reproduction of intellectual property in the presence of demand network externalities. Journal of Industrial Economics 42:155-66.

Van den Bulte, Christophe, and Gary L. Lilien. 1999. Integrating models of innovation adoption: Social contagion, utility maximization, and hazard models. Working Paper no. 99-021, Pennsylvania State University, Department of Marketing.

Van Huyck, John B., Raymond C. Battalio, and Richard O. Beil. 1990. Tacit coordination games, strategic uncertainty and coordination failure. American Economic Review 80:234-48.

Williams, Sam. 2002. Profits from piracy. Available at http://salon.com/tech/feature/2002/09/26/ piracy_unlimited/print.html, September 26. 
Copyright of Journal of Business is the property of University of Chicago Press and its content may not be copied or emailed to multiple sites or posted to a listserv without the copyright holder's express written permission. However, users may print, download, or email articles for individual use. 